linkages come into existence by the formation of divinylbenzene bridges. Such a cross-linked system is no longer dispersible in solvents, although if not too many cross-linkages are formed the material is capable of swelling. If the ratio of styrene to divinylbenzene is as high as 50,000 to 1 , the product is highly extensible but insoluble, whilst if it be as low as 1,000 to 1 the product shows scarcely any sign of swelling at all.

It is clear that the properties of the polymers and condensation products may be altered to a most extraordinary extent by the addition of suitable materials even in subanalytical amounts.

ERIC K. Rideal.

\title{
Maintenance of Life in Isolated Animal Organs
}

$\mathrm{T}^{\mathrm{H}}$ HE preliminary announcement of the work of Carrel and Lindberg on the maintenance of life in isolated organs, to which reference has already been made in our columns ${ }^{1}$, can now be amplified with further details of their method, from which an idea can be obtained of how the difficulties involved have been surmounted $^{2}$. The organs are removed with complete aseptic and antiseptic precautions from an animal that has just been killed by bleeding under anæsthesia; adult cats or fowls are generally used. With the organ are removed the surrounding tissues, arteries, veins, nerves and lymph vessels : for example, in the case of the ovary, with the organ itself are removed the Fallopian tube, and a flap of peritoneum and connective tissue containing the ovarian artery. During the operation the abdominal cavity and the organ are protected with gauze pads soaked in Dakin's solution.

The organ is introduced into the culture chamber protected by a sheet of 'cellophane' and the artery is connected with the cannula of the apparatus ; the chamber is closed by a rubber stopper and sealed with a cellulose acetate cement. A sterile pulsating circulation is maintained through the organ by the apparatus; different culture media have been used for this purpose. In order that the supply of glucose and bicarbonate may be sufficient for several days, the volume of the medium must be about 2,000 times greater than that of the tissues. For example, a cat's thyroid which weighs $85-110 \mathrm{mgm}$. requires about 230 c.c. of fluid. The apparatus may be used with 200 900 c.c. of medium. The latter may be bloodserum, diluted with Tyrode's solution, and containing sometimes hæmoglobin, or an artificial growth-activating solution, containing proteinsplit products, hæmin, cysteine, insulin, thyroxine, glutathione, vitamins $\mathrm{A}$ and $\mathrm{C}$, blood-serum, ete. A small amount of phenol red must be added, as indicator of the metabolic activity of the organ, as well as of the occurrence of bacterial infection. The medium is under an atmosphere of 40 per cent oxygen and 3-4 per cent carbon dioxide, the remainder being nitrogen, and is kept well aerated.
The apparatus is kept in an incubator at $37^{\circ}$ $38^{\circ} \mathrm{C}$. and is so designed that the organ and perfusion fluid can be continuously observed. The circulation is started about an hour after the death of the animal ; the number of pulsations is usually about 60 per minute, the systolic pressure being $120 \mathrm{~mm}$. mercury and the diastolic $60 \mathrm{~mm}$. mercury. Rate and pressure can be varied at will.

The organs used in the apparatus so far have been the thyroid gland, the ovary, the suprarenal gland, the spleen, heart and kidney. In the case of the thyroid, three different media have been used as perfusion fluids ; with diluted serum or an artificial medium containing amino-acids, the volume of the gland decreased and the follicles remained full of colloid, even after twenty-one days. With the medium containing peptones the glands grew and doubled their weight in three to four days; the consumption of sugar was increased more than three times. Histologically, there were observed either a disordered epithelial proliferation with disappearance of the colloid or an increase in the volume of the cells and in the number of follicles, with production of colloid. In an experiment with an ovary perfused with a growth-promoting medium, the weight of the organ increased from $90 \mathrm{mgm}$. to $284 \mathrm{mgm}$. in five days and three corpora lutea developed. Growth was, however, accompanied by disorganisation; there was a luxuriant and disordered proliferation of the stroma and of the epithelial cells.

These experiments show that an entire organ can be kept alive in vitro, increasing in size and weight, due to the appearance of new cells and tissues. The method should have great value in the study of different organs, especially those having an 'internal secretion', and further reports by Carrel and Lindberg will be awaited with interest. If it is permissible to speculate about the future, will it be possible for the chemist to utilise isolated perfused organs for the manufacture of their specific secretions in unlimited quantities, in cases in which a chemical synthesis is difficult or has not yet been achieved?

${ }^{1}$ NATURE, 135, 1066 ; 1935.

Science, 621, June 21, 1935. C.R. Acad. Sci., 14, July 1, 1935. 